\title{
Medición y Análisis de Anuncios Publicitarios en Televisión con base en las Herramientas Seguidor-de-Visión y Lector-de-Rostro (EyeTracking y FaceReader)
}

\author{
Jairo A. Rodas, y Luz A Montoya-Restrepo \\ Univ. Nacional de Colombia, Facultad de Minas, Dpto. de Ingeniería de la Organización, Medellín - Colombia \\ (e-mail: jarodas@unal.edu.co; lamontoyar@unal.edu.co)
}

Recibido May. 24, 2018; Aceptado Jul. 30, 2018; Versión final Ago. 11, 2018, Publicado Abr. 2019

\begin{abstract}
Resumen
El objetivo de este trabajo fue la determinación de una metodología para la evaluación de un video comercial, desde la identificación del nivel de impacto, estados emocionales y patrones de atención de los consumidores. Se establecieron elementos a analizar, propios de la experiencia y se estudiaron a través de herramientas de neuromarketing, tales como el Seguidor-de-Visión y el Lector-de-Rostro (EyeTracking y FaceReader), y se complementan con elementos de la investigación tradicional de mercados. El estudio presenta las principales variables para la aplicación de esta metodología, donde se analizan e integran las respuestas del consumidor. Basado en los resultados del estudio, se concluye que es posible definir elementos que generan impacto atencional, cognitivo, emocional y de argumentación racional, a través de un proceso sistemático.
\end{abstract}

Palabras clave: neuromarketing; publicidad; comportamiento del consumidor; eyetracking; facereader

\section{Measurement and Analysis of Television Commercials based on the Computer Tools EyeTracking and FaceReader}

\begin{abstract}
The objective of this paper was the determination of a methodology for the evaluation of a commercial video, from the identification of the level of impact, emotional states and patterns of attention of consumers. The elements of experience to be analyzed were established and were studied through neuromarketing tools, such as EyeTracking and FaceReader, and are complemented by elements of traditional market research. The study presents the main variables for the application of this methodology, where consumer responses are analyzed and integrated. Based on the results of the study, it concluded that it is possible to define elements that generate attentional, cognitive, emotional and rational argumentation impact, through a systematic process.
\end{abstract}

Keywords: neuromarketing; advertising; consumer behavior; eye tracking; facereader 


\section{INTRODUCCIÓN}

En la actual dinámica empresarial, el mercadeo ha evolucionado debido a la importancia que se le ha atribuido a la experiencia del consumidor, donde, y según Miller y Washington (2012) las experiencias de los clientes integradas a las actividades son más memorables; lo cual puede crear una conexión más significativa, afectiva y de valor de marca con los consumidores, impactando positivamente la construcción de relaciones duraderas en el tiempo. Desde hace algunos años, la comunicación y la publicidad desempeñan un papel fundamental en la estrategia, y como lo plantea Khan (2014) cumplen tres funciones básicas, las cuales son: proporcionar información y asesorar, persuadir al público objetivo e invitarlo a actuar en momentos específicos relacionados con la toma de decisiones de compra y consumo; a través de un conjunto de mensajes y elementos que generan emociones, sensaciones, sentimientos y argumentos racionales, que pueden evocar, ser base para la generación, o participar de una experiencia.

A partir de lo anterior es necesario garantizar el diseño y la evaluación adecuada de las piezas publicitarias, donde se identifique qué elementos limitan el cumplimiento de los objetivos; y cuales aportan valor a la táctica definida; en la que se determinen los momentos y elementos de mayor atención e impacto, la forma en qué un consumidor observa y analiza, y las emociones específicas producidas por un conjunto de estímulos que impactan uno o varios sentidos, los cuales son procesados y generan una respuesta conductual específica, relacionada con la compra. Para el análisis y la evaluación de lo planteado anteriormente, es necesario tener en cuenta a Bakardjieva y Kimmel (2017), los cuales manifiestan que los enfoques y las técnicas continúan evolucionando junto con los desarrollos en tecnologías emergentes, proporcionando nuevas oportunidades para que los investigadores y los profesionales de mercadeo obtengan información sobre la toma de decisiones y el comportamiento, es decir, para estudios relacionados con el conocimiento del consumidor.

Se trabaja tanto técnicas tradicionales de investigación de mercados, como nuevas técnicas, las cuales incluyen el uso de herramientas tecnológicas y de lectura cerebral, tomadas de las neurociencias y aplicadas al mercadeo bajo el concepto de neuromarketing, del cual Salazar (2011) presenta que, este permite analizar la actividad cerebral, obteniendo datos extras, teniendo en cuenta que son muchos los estímulos que influencian en el comportamiento del evaluado, lo que brinda la oportunidad de interpretar dichas conductas, y de esta manera hacer una breve descripción o predicción de futuros comportamientos de acuerdo a sus sentimientos, emociones y actos. Particularmente, en esta investigación se propone como objetivo general establecer una metodología para la evaluación de un video comercial de televisión, desde la identificación de elementos de impacto, estados emocionales y patrones de atención de los participantes.

Para cumplir con el objetivo propuesto se desarrolla una investigación de tipo cualitativo de naturaleza exploratoria, donde participan 20 mujeres, las cuales son expuestas a la proyección de un comercial de televisión y son evaluadas con la utilización de dos herramientas tecnológicas del neuromarketing: el Eye tracking; el cual es conceptualizado por Zurawiki (2010), como un instrumento por medio del cual es posible analizar la forma en que se lee, la distribución y el tiempo de mirada, y por último pero no menos importante, la dilatación de la pupila. El seguimiento ocular ayuda a observar la forma en que la impresión influye en la atención del sujeto y en el procesamiento cognitivo; y el FaceReader, el cual es un software de reconocimiento facial, el cual permite analizar en tiempo real las expresiones del consumidor y asociarlas a emociones específicas.

Al revisar la literatura sobre el tema, se destaca que desde hace unos 20 años se están utilizando herramientas de las neurociencias y posteriormente han sido aplicadas al análisis de las estrategias de mercadeo. Algunos investigadores como Bojko (2006), el cual trabajó sobre la página web de la Sociedad Estadounidense de Oncología Clínica, donde a través del Eye Tracking se evaluó la participación de 12 miembros de la ASCO, quienes navegaron en dos páginas web con diferente diseño; se analizaron tareas como las ventanas más usadas de acuerdo a la ubicación en la interfaz y el tamaño de los gráficos, con el fin de determinar cuál diseño ofrecía mayores beneficios al usuario. Otro de los trabajos fue el de Marcos y González (2010), quienes convocaron 58 participantes con el objetivo de comprobar si la intención de las consultas afecta a la forma en que el usuario explora visualmente la página de resultados de un buscador, se desarrolla el experimento con las plataformas de Google y Yahoo.

Khushaba et al (2013) presentan en su trabajo el uso del Eye Tracking y lo complementan con el EEG (electroencefalograma), donde se observó y evaluó la actividad cortical de las diferentes regiones cerebrales, de 18 participantes, en el proceso de elección de galletas, teniendo en cuenta la forma, el sabor y los ingredientes; con respecto al trabajo sobre la variable promoción, Hernández y Muñoz (2015) trabajaron con la participación de 60 personas para analizar el impacto de la publicidad en línea, para banners estáticos como dinámicos, trabajando tanto imágenes como textos, utilizando la tecnología del Eye Tracking. 
Con respecto a los experimentos descritos anteriormente se evidencia, que aunque logran el objetivo que se plantean, no se presenta una medición a la experiencia desde diferentes perspectivas, se limita a las técnicas de medición, y no al diseño y desarrollo de una metodología; complementando lo anterior y tal y como lo plantea Schmitt (2006), cuando se trabaja el tema del impacto a los sentidos en el mercadeo, es de mencionar, que aunque se presenta una amplia gama de métodos de investigación, no se encuentran vinculados con ninguna corriente metodológica; es decir, los métodos son ecléticos; a lo que Moral y Fernández agregan (2012), el marketing experiencial no se encuentra circunscrito a una metodología de investigación concreta, sino que adopta instrumentos amplios y diversos.

\section{METODOLOGÍA}

Se desarrolló una investigación donde se utilizaron dispositivos tecnológicos (Eye Tracking y FaceReader), y se definieron y evaluaron los elementos, mensajes y estímulos producidos por un comercial de televisión para la categoría de cremas faciales para mujeres; es de aclarar que este puede ser aplicado para cualquier sector, categoría y producto.

\section{Objetivo}

El objetivo general se determinó como: establecer una metodología para la evaluación de un video comercial, desde la identificación de elementos de impacto, estados emocionales y patrones de atención de los consumidores. Para cumplir con este objetivo, primero, identificando los elementos a evaluar, se recolectaron los datos a través de los dispositivos electrónicos, y se realizaron los análisis pertinentes para obtener respuestas, que se integran en la medición de la experiencia. Cabe resaltar que para la construcción de la metodología se utilizaron diferentes conceptos, para la validación el trabajo.

\section{Elementos analizados en la investigación}

El sentido visual representa uno de los elementos más importantes del estudio de la variable promoción, y de acuerdo a Baptista et al (2012), esto ocurre debido a su papel fundamental en el reconocimiento y recuerdo de la imagen, la publicidad, los empaques, los productos, los símbolos, las marcas, los logotipos, la ubicación de los productos, los colores y demás aspectos clave. Otra de las variables es el aspecto auditivo, sobre el cual, estos autores mencionan que para el mercadeo, las diversas cualidades de los sonidos (tonos, ritmos, intensidades, volúmenes, entre otros) representan elementos primordiales en los procesos que implican la transmisión de emociones al cliente, el reconocimiento de marcas, posicionamiento, publicidad, venta personal y mercadeo directo.

Para el desarrollo del trabajo y el análisis del comercial se definió de manera previa un conjunto de elementos de dos tipos: a nivel visual, las personas o protagonistas del comercial, el entorno o atmósfera donde se desarrolla los hechos (colores, objetos, fondo, iluminación), los textos que se leen, la imagen y marca, el producto; y a nivel auditivo, las palabras o guion de los protagonistas, los sonidos, la música. Además de los establecidos, se trabaja sobre el análisis de los elementos de impacto general encontrados durante los experimentos.

\section{Métodos de obtención de datos}

Para la obtención de datos y la evaluación del comercial de televisión, cuya duración es de 60 segundos, el cual se encuentra disponible en el siguiente enlace https://goo.gl/EwV6Bu; se utiliza el EyeTracking y el FaceReader (herramientas del neuromarketing) de manera simultánea en 20 participantes del género femenino (las cuales firmaron el consentimiento informado, donde se planteaban los objetivos y la logística técnica y operativa del experimento), el primero de los dispositivos mide estados de atención e impacto, y el segundo presenta emociones específicas: además previo a la ejecución de las pruebas de laboratorio, se sometió el proyecto al Comité de Ética de la Universidad Nacional de Colombia, el cual lo aprobó.

Los sentidos estimulados por los elementos del comercial fueron la escucha y la vista (como se describió anteriormente). Los resultados de cada uno de los experimentos fueron extraídos de manera sincronizada, lo que permitió el análisis paralelo e integral. Es de resaltar que bajo la metodología planteada, es posible, identificar el impacto de la pieza publicitaria y de los elementos específicos que la conforman.

\section{Procedimiento y análisis}

Para el desarrollo del trabajo experimental se siguió un procedimiento, que se divide en tres etapas:: la primera fue de planeación, comenzando con la detección de la necesidad de información, la cual para este trabajo destaca la importancia del análisis del impacto de las piezas publicitarias, y la utilización de dispositivos 
para su medición, como lo plantean Gómez et al (2016) las TIC ( tecnologías de la información) han cambiado la forma de vida de los seres humanos, impactando la producción y comercialización de las empresas, en este caso, en lo relacionado con el diseño y la evaluación publicitaria; con respecto a las hipótesis, se tiene que, es posible a través de herramientas conceptuales y tecnológicas medir los resultados de piezas publicitarias, basadas en experiencias multi sensoriales. Es de aclarar que aunque las pruebas se desarrollaron para cremas faciales y la medición se realiza para la ciudad de Medellín, Colombia; no se limita el alcance, pues este trabajo puede ser adaptado a otros sectores, categorías, públicos y territorios geográficos.

La segunda etapa fue la del diseño, para la cual se definió que para el desarrollo de este trabajo, se propusiera la investigación exploratoria, y como se mencionó anteriormente se realizó a través del FaceReader y el Eye tracking, midiendo las respuestas de 20 mujeres (diseño no probabilístico), mayores de 25 años, con nivel de escolaridad profesional (en capacidad económica de incluir este producto en sus compras), y que dentro de sus hábitos tuvieran el consumo frecuente de cremas faciales; con esta segmentación se buscó uniformidad de la muestra para evitar sesgos en la misma y hacer que los resultados fueran comparables, además era importante que las participantes usaran frecuentemente este tipo de productos para la formación de criterio personal sobre lo analizado. Es de resaltar que previamente se realizó calibración del Eye Tracking y pruebas con el FaceReader. En esta etapa se definieron las áreas de interés del comercial de televisión sobre las cuales se enfoca el análisis, se establece una división por escenas, para identificar cambios en las respuestas de las participantes y elementos de influencia, y se realiza definición de frases para presentar un análisis semántico de mensajes transmitidos y recibidos vía auditiva.

Finalmente, en la etapa de implementación y análisis se parte de la información recolectada y se establecieron una serie de conclusiones, extraídos de la integración y conceptualización de los datos obtenidos. En este aparte se identificaron elementos de mayor impacto, y se establecen las respuestas de naturaleza de atención, cognitiva y emocional.

\section{RESULTADOS Y DISCUSIÓN}

Complementando lo que presentan los investigadores del tema, los resultados pueden dividirse en resultados del proceso y de la argumentación racional, en este último caso se abalizó si los elementos evaluados son importantes o no para el participante de la experiencia, y confrontar los resultados con la evaluación a través de herramientas de neuromarketing. Por esto, la combinación de técnicas de investigación (con herramientas tecnológicas y no tecnológicas) permite comprender mejor la percepción del consumidor con respecto a lo evaluado; e incluso en algunos casos los resultados de una técnica pueden complementar y explicar los resultados de otra.

En una misma experiencia se pueden presentar diferentes emociones, estados de atención e impacto y elementos que generan diferentes respuestas, por lo que se debe trabajar sobre áreas de interés (definición de elementos o conjunto de elementos), en busca de la identificación de estímulos que generan respuestas positivas, neutras y negativas en el consumidor. A esto se le suma, que cuando se trabaja en la medición de las experiencias sensoriales se debe tener en cuenta que funcionan bajo el principio de la crosmodalidad, es decir, los sentidos no trabajan de manera independiente; pero la percepción y la creación de imágenes mentales se producen de manera integral.

\section{Resultados de la medición con Eye Tracking y FaceReader}

A continuación en la Tabla 1 se presentan los resultados más importantes en la medición de los principales elementos que conforman el comercial de televisión, es de aclarar que el segmento uno (denominado en la investigación como intención comunicativa), hace referencia al intervalo de tiempo del segundo uno al siete; el dos (denominado validación por especialista), del segundo siete al 14; el tres (efectividad por validadores), del segundo 14 al 19; el cuatro (explicación técnica), del segundo 19 al 23; el cinco (efecto dramatizado), del segundo 23 al 32; el seis (casos de éxito), del segundo 32 al 37; el siete (modos de uso), del segundo 37 al 41 ; el ocho (recomendación de la línea), del segundo 41 al 48; y el nueve (promesa del producto), del segundo 48 al 60.

Para la interpretación de estos resultados es necesario exponer, que los elementos pudieron haber sido percibidos por las consumidoras de diferente manera en cada una de las escenas, ya que podían complementarse con otros e incluso el contexto podía verse modificado por estímulos de diferente naturaleza; en la Tabla 1, es posible analizar estas métricas, donde se presenta el índice de visualización a partir del Eye Tracking, y el tipo de respuesta emocional, la cual puede ser positiva, negativa o neutra. 
La felicidad y la tristeza fueron las emociones específicas que predominaron en las consumidoras del experimento, teniendo mayor participación la segunda. En la Tabla 2, se presentan las emociones predominantes (tanto positivas como negativas) generadas a partir del FaceReader, para cada uno de los segmentos de tiempo establecidos previamente para el comercial de televisión. Para identificar la magnitud de estas, es necesario se trabaje de manera paralela con la Tabla 1. Luego se presenta una serie de elementos conceptuales, teóricos y prácticos que complementan lo presentado anteriormente

Tabla 1: Resultados Eye Tracking y FaceReader para el análisis de la visualización de las participantes

\begin{tabular}{|l|l|c|c|c|c|}
\hline \multicolumn{5}{|c|}{ Impacto total integración áreas de interés en el comercial } \\
\hline \multirow{2}{*}{ Áreas de interés } & \multicolumn{1}{|c|}{ Segmento } & $\begin{array}{c}\text { Índice de } \\
\text { Visibilidad }\end{array}$ & \multicolumn{3}{c|}{ Respuesta emocional } \\
\cline { 3 - 6 } & & $96 \%$ & $36 \%$ & $64 \%$ & $0 \%$ \\
\hline Modelo & $1,2,4,5,6,7,8$ y 9 & $78 \%$ & $22 \%$ & $49 \%$ & $29 \%$ \\
\hline Dermatóloga & $2,3,6$ y 7 & $58 \%$ & $5 \%$ & $75 \%$ & $20 \%$ \\
\hline Producto & $2,3,6,7$, y 9 & $19 \%$ & $11 \%$ & $0 \%$ & $89 \%$ \\
\hline Descriptor inferior & $1,2,3,4,5,6,7,8$ y 9 & $70 \%$ & $100 \%$ & $0 \%$ & $0 \%$ \\
\hline Componentes & 4 & $4 \%$ & $0 \%$ & $0 \%$ & $100 \%$ \\
\hline Expresión aclara & 5 & $83 \%$ & $33 \%$ & $67 \%$ & $0 \%$ \\
\hline Casos de éxito & 6 & & & & Negativa \\
\hline
\end{tabular}

Tabla 2: Emociones generadas por segmento

\begin{tabular}{|l|c|c|}
\hline \multicolumn{3}{|c|}{ Emociones específicas generadas } \\
\hline Segmento & $\begin{array}{c}\text { Emoción negativa } \\
\text { predominante }\end{array}$ & $\begin{array}{c}\text { Emoción positiva } \\
\text { predominante }\end{array}$ \\
\hline Uno & Tristeza & Felicidad \\
\hline Dos & Tristeza & Felicidad \\
\hline Tres & Desprecio & Felicidad \\
\hline Cuatro & Tristeza & Felicidad \\
\hline Cinco & Rabia & Felicidad \\
\hline Seis & Sorpresa & Felicidad \\
\hline Siete & Rabia & Felicidad \\
\hline Ocho & Desprecio & Sorpresa \\
\hline Nueve & Tristeza & Felicidad \\
\hline
\end{tabular}

Atención, percepción e impacto de los elementos

El individuo recibe señales sensoriales provenientes del exterior e interior de su organismo (Estévez et al, 1997); sin embargo, la cantidad de información entrante excede la capacidad del sistema nervioso para procesarla en paralelo, por lo que se hace necesario un mecanismo neuronal que regule y focalice el organismo (Mesulam, 1985), seleccionando y organizando la percepción, y permitiendo que un estímulo pueda dar lugar a un 'impacto'; es decir, que pueda desarrollar un proceso neural electroquímico, este mecanismo es conocido como la atención. Cuando se menciona el término percepción, se plantea que es un análisis rápido de los estímulos a varios niveles, donde se resalta a Craik y Lockhart (1980), los cuales plantean que en los primeros, se realiza el estudio de determinadas características físicas o sensoriales como las líneas, los ángulos, el brillo, el tono y la sonoridad; a diferencia de los demás niveles, donde se relacionan con la comparación entre las entradas y las abstracciones almacenadas a partir del aprendizaje.

Al analizar los resultados obtenidos para este caso, se tiene que los elementos con mayor visualización extraída con base en el Eye Tracking, fueron las personas, representadas en la influenciadora social (modelo y presentadora) y la especialista en salud (dermatóloga), es de aclarar y como se presentará más adelante, alta visualización, no significa necesariamente algo positivo. Por otro lado se tiene que, el producto (crema anti manchas) tuvo una visualización media $(58,6 \%)$, lo que se puede explicar a partir del papel que desempeña este en la mayoría de las escenas o fragmentos (fondo), bajo el concepto teórico de percepción de figura y fondo, además este, no logra enganchar a las participantes en la primera visualización, donde se presenta bajo impacto en elementos de primer orden, lo que no activa el proceso de una observación más profunda (cognitiva, es decir, de segundo orden). 


\section{Emociones específicas}

Las emociones y las sensaciones generalmente están ligadas a las percepciones, que determinan la visión que los seres humanos tienen del mundo, y de esa visión se derivan comportamientos, muchos de los cuales son de suma utilidad para las organizaciones. (Baptista et al, 2010). Es por esto que la comprensión de los elementos mencionados anteriormente, es vital para el desarrollo de estrategias y acciones de mercadeo.

Por otro lado, tal y como lo plantean Gómez \& Mejía (2012) además de la percepción, el uso del estímulo de los sentidos influye sobre las emociones y la memoria del ser, lo que permite lograr en el largo plazo relaciones afectivas y mayor permanencia de la marca; reflejándose en temas como la fidelización y la recomendación, el relacionamiento, la recompra, y el posicionamiento y recordación de marca, productos y servicios. Las emociones tienen tres enfoques funcionales, que según Reeve (1994) se dividen en: adaptatitvo, cuyo fin es preparar al organismo para que ejecute eficazmente la conducta exigida por las condiciones ambientales; el segundo permite movilizar la energía necesaria para ello, y por último dirigir la conducta (acercar o alejar) hacia un objetivo determinado.

Por medio del FaceReader es posible, a través de la lectura de micro músculos faciales junto a una cámara de video, identificar las emociones específicas experimentadas por un participante al estar expuesto a un estímulo natural o artificial, y en el caso del comercial, al ser impactados los sentidos de la vista y el sistema auditivo, por un conjunto de imágenes y sonidos. Las emociones que son posible determinar con el FaceReader son: la felicidad, la sorpresa, el disgusto, el miedo, el desprecio, la tristeza, la rabia y el neutral. Esta herramienta complementa al Eye Tracking, pues permite profundizar, y presentar la explicación de cuando una visualización alta se está danto, o lo que se está percibiendo con que emoción se asocia. Estos dispositivos son de gran importancia, especialmente en la evaluación publicitaria actual, donde, las experiencias, son multi sensoriales, permitiendo la comprensión y el establecimiento de impactos deseados y no deseados en el diseño de la pieza publicitaria.

En el experimento de neuromarketing se evidencia, que aunque algunos elementos tienen alta visualización en la extensión de la pieza publicitaria, como es el caso de la modelo o presentadora, se tiene que por ejemplo en los primeros siete segundos sólo se evaluó otro elemento adicional (el descriptor), el cual no tuvo visualización; y al analizar las emociones específicas del segmento, se identifica que la predominante fue la tristeza. Complementando lo anterior, se identifica que las participantes al evaluar el segmento denominado efecto dramatizado, presentado en el intervalo de tiempo comprendido entre los segundos 23 y 32 , perciben una experiencia negativa, lo cual es ratificado en la argumentación racional en los resultados presentados en una investigación de mercados de naturaleza descriptiva, donde se trabaja sobre la misma pieza publicitaria.

\section{Análisis semántico}

Los elementos del lenguaje cumplen funciones específicas en el diseño publicitario, a través de los mensajes utilizados y transmitidos al público objetivo, sea por medios masivos o no masivos; y es que como lo presenta González (2008), desde los primeros inicios de la humanidad, el hombre buscaba un medio para registrar sus emociones, sentimientos, situaciones y hechos; por lo que surgió entonces, el lenguaje. Cualquier lengua humana tiene una estructura gramatical en la que las unidades fónicas (señalizadoras) se combinan entre sí para producir un significado específico. En el lenguaje humano es imprescindible que el hablante relacione unos sonidos con un significado y que a su vez ese significado sea percibido y comprendido por las demás personas que comparten la misma lengua.

En el comercial se presentan diferentes tipos de lenguaje, uno de los más relevantes es el lenguaje hablado, el cual está representado en las expresiones de las personas que lo protagonizan. A través de la metodología de análisis semántico y con el apoyo de la tecnología (FaceReader), es posible identificar la percepción ante las principales frases emitidas y recibidas por las participantes del estudio. Después de este análisis y partiendo de los datos recolectados, se tiene que, frases como "ocho de cada diez mujeres notaron la reducción" o "está dermatológicamente probada", es decir, relacionadas con el tema efecto o resultados, produjeron alto nivel de rabia, lo cual se debe a que las personas no creen en este tipo de datos (resultado de una investigación descriptiva y concluyente), especialmente para este tipo de producto, para el cual se debe confiar, para posteriormente ser consumido.

\section{Medición y análisis de anuncios publicitarios en televisión}

En la Fig.1, se presenta la metodología para la medición y análisis de anuncios publicitarios en televisión, y se describe a continuación: para comenzar, es necesario identificar el objetivo de la pieza publicitaria, es decir, qué se pretende transmitir al receptor con el mensaje del mismo; luego se establece desde la argumentación racional, sí los elementos que conforman el comercial son los adecuados (personas, objetos, atmósfera, 
guion, colores, sonidos); para posteriormente realizar la evaluación de estos, a través de herramientas del neuromarketing como el FaceReader y el Eye Tracking, estableciendo de manera previa las escenas de la pieza dinámica a trabajar y lo que se evaluará en cada una de ellas, es decir, las categorías de análisis y los elementos (definición de áreas de interés); y finalmente se extraen las frases mencionadas por los protagonistas con las que se esperaba generaran mayor impacto para ser trabajadas desde el análisis semántico.

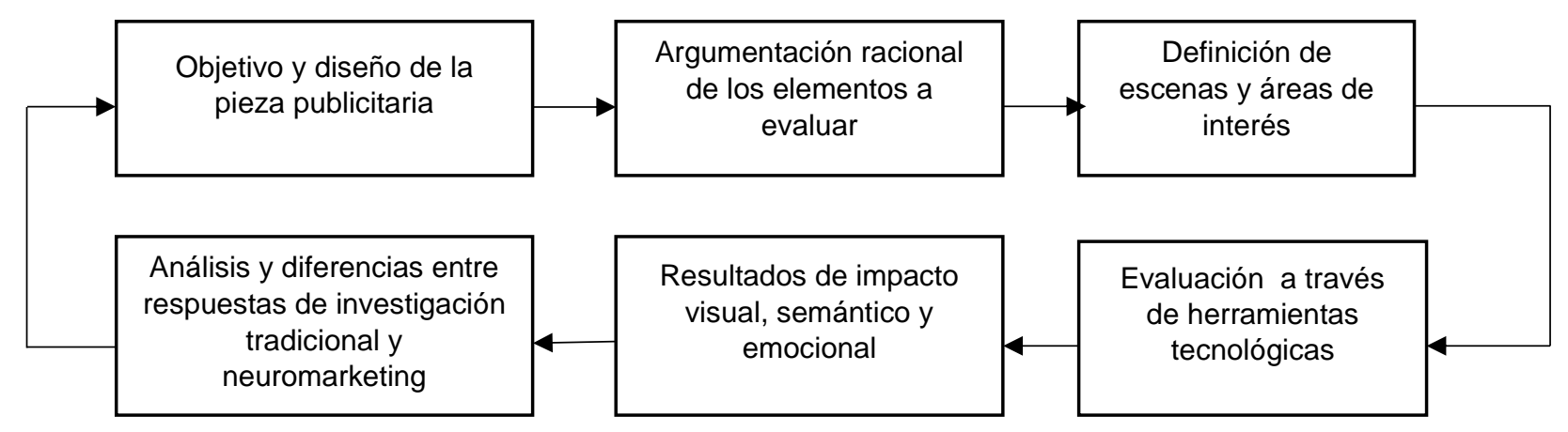

Fig. 1: Metodología para el análisis y medición de comerciales de televisión con base en neuromarketing

Luego se obtienen los indicadores de visualización e impacto a través del seguimiento ocular, y las emociones específicas a través del FaceReader, las cuales son analizadas y complementadas con información previa de argumentación racional (de la investigación de mercados tradicional) del consumidor, influenciados por factores personales, culturales, sociales y psicológicos, relacionados con el proceso de toma de decisiones de compra, para encontrar explicaciones y diferencias en los diferentes tipos de respuestas, buscando integralidad en el análisis.

Es de aclarar que este proceso puede ser aplicado en la etapa de diseño, permitiendo mejoras antes de la emisión en los medios (es por esto que este proceso presenta una estructura cíclica), disminuyendo el riesgo de no tener los resultados esperados; y en la etapa de auditoría de las acciones de mercadeo para medir impacto, proceso que ha sido calificado como subjetivo a lo largo de los años, especialmente en lo que respecta a los medios de comunicación masiva.

\section{DISCUSION FINAL}

A partir de esta investigación teórico-práctica, se evidencia un conjunto de aportes al conocimiento en el campo trabajado, dónde se complementa lo planteado en los últimos años por algunos investigadores, partiendo de la importancia de la comprensión de los procesos de decisión relacionados con la compra, la elección de un producto y la experiencia del consumidor, al presentarse estímulos internos y externos, derivados de acciones de marketing como los de la promoción. Para medir el impacto de las piezas publicitarias y de comunicación es necesario trabajar bajo un proceso, donde los estímulos de diferente naturaleza generan respuestas específicas, que pueden relacionarse, presentar diferencias e incluso argumentar algunas de otro tipo. El estudio de estos fenómenos no se debe realizar de manera aislada, a partir de la aplicación de diferentes técnicas; pues el ser humano es integral y responde a través de la razón, de las emociones, de las sensaciones, y de los conocimientos previos, entre otros; generándose una respuesta que las agrupa, y produciendo un efecto en la elección y en la toma de decisiones.

En cuanto a las experiencias, y donde el consumidor recibe continuamente información, es vital el análisis de los cambios en los niveles de atención, estados cognitivos y emocionales. El ser humano no presenta constancia en sus sensaciones, es decir, durante una experiencia pueden producirse diferentes respuestas y percepciones, a partir de la activación de diferentes sentidos (las experiencias son multisensoriales) y del impacto de diferentes elementos; y es por eso que la metodología planteada en este artículo no sólo permite evaluar la experiencia publicitaria en conjunto, también permite identificar la importancia de elementos específicos desde el impacto, la atención y el nivel de visualización, la empatía con los protagonistas de la pieza en una escena o fragmento y la función que cumplen dentro del comercial.

Finalmente, con respecto a la percepción cabe resaltar que los sentidos generan la primera idea y percepción sobre el mundo, pero otras variables como la razón, los factores sicológicos, los factores sociales, factores culturales, entre otros, permiten una mejor comprensión de lo que sucede en la mente del consumidor. 


\section{CONCLUSIONES}

Con base en los resultados de este estudio, y el análisis teórico conceptual, se pueden extraer las siguientes tres conclusiones principales: 1) es posible medir los resultados de una pieza publicitaria a través del complemento, integración y argumentación entre herramientas de investigación tradicional y del neuromarketing; 2) la guía presentada en este trabajo permite de manera integral analizar elementos que generan impacto atencional, cognitivo, emocional y de argumentación racional (proveniente de factores sociales, personales, culturales y psicológicos), que pueden ser resultado de experiencias previas; y 3) la medición de una pieza publicitaria con dispositivos tecnológicos, puede realizarse en la etapa de diseño (antes de ser transmitida al público objetivo) o en la etapa de evaluación (posterior a ser emitida en los medios de comunicación).

\section{AGRADECIMIENTOS}

Los autores agradecen al Departamento Administrativo de Ciencia, Tecnología e Innovación (Colciencias) y a la Universidad Nacional de Colombia por la gestión de los recursos financieros para llevar a cabo los diseños experimentales de este proyecto investigativo, enmarcado en la Tesis Doctoral denominada: Desarrollo Metodológico para la Medición y el Análisis de Estrategias basadas en Sensory Marketing en el sector Cosmético para las Mujeres del municipio de Medellín.

\section{REFERENCIAS}

Bakardjieva, E. y A.J. Kimmel, Neuromarketing Research Practices: Attitudes, Ethics, and Behavioral Intentions, doi: 10.1080/10508422.2016.1162719, Ethics \& Behavior, 27(3), 179-200 (2017)

Baptista, M.V., M. León y C. Mora, Neuromarteking: Conocer al Cliente por sus Percepciones (Neuromarketing: know customers through their perceptions), Tec. Empresarial, 4 (3), 9-19 (2012)

Bojko, A., Using Eye Tracking to Compare Web Page Designs: A case study, Journal of Usability Studies, 1(3), 112120 (2006)

Craik, F. y R. Lockhart, Niveles de Procesamiento: un Marco para la Observación sobre la Memoria, Estudios de Psicología, Número 2, Universidad de Toronto (1980)

Estévez, A., C. García y C. Junqué, La Atención: una Compleja Función Cerebral, Revista de Neurología, 25(148), 19891997 (1997)

Gómez, E., J. Pérez y J. Ramírez, Sistema de Información Agrícola para la disminución de Brechas entre Oferta y Demanda-AGROCRAFT, doi: 10.4067/S0718-07642016000300020, Inf. Tecnol, 27(3) 3, $215-220$ (2016)

Gómez, R.C., y J., Mejía, La gestión del Marketing que conecta con los Sentidos, doi: 10.21158/01208160.n73.2012.592, Revista Escuela de Administración de Negocios, (73), 168-183 (2012)

González, J., La Lógica del Lenguaje, Revista Pensamiento Americano, 1 (1), (2013)

Hernández-Méndez, J. y F., Muñoz-Leiva, What type of Online Advertising is most effective for eTourism 2.0? An Eye Tracking Study based on the Characteristics of Tourists, doi: 10.1016/j.chb.2015.03.017, Computers in Human Behavior, 50, 618-625 (2015)

Khan, M., The Concept of'Marketing mix'and its elements (a conceptual review paper), International Journal of Information, Business and Management, 6 (2) 95 (2014)

Khushaba, R.N., C., Wise y otros cuatro autores, Consumer Neuroscience: Assessing the Brain Response to Marketing Stimuli using Electroencephalogram (EEG) and Eye Tracking, doi: 10.1016/j.eswa.2012.12.095, Expert Systems with Applications, 40(9), 3803-3812 (2013)

Marcos, C. y C., González-Caro, Comportamiento de los Usuarios en la Página de Resultados de los Buscadores. Un Estudio basado en Eye Tracking, El Profesional de la Información, 19(4), 348-358 (2010)

Mesulam, M., Attention, Confusional states, and Neglect, Principles of Behavioral Neurology, 125-168 (1985)

Miller, R.K. y K., Washington, Event and Experiential Marketing, In R. K. Miller, \& K. Washington (Eds.), Consumer Behaviour, Atlanta GA: Richard K. Miller \& Associates, 427-429 (2012)

Moral, M. y M., Fernández, Nuevas Tendencias del Marketing: el Marketing Experiencial, Revista Entelequia, 14, 237 (2012)

Salazar, C., La Neurociencia del Consumidor como horizonte de Investigación, Conceptos y Aplicaciones. Un enfoque Paradigmático, Universidad \& Empresa, Julio-Diciembre, 143-166 (2011)

Schmitt, B., Experiential Marketing: Cómo conseguir que los Clientes identifiquen en su Marca sensaciones, Sentimientos... Relaciones, Ediciones Deusto, Bilbao, Spain, (2006)

Zurawicki, L., Neuromarketing, Exploring the Brain of the Consumer, Springer-Verlag Berlin Heidelberg, pp. 42 (2010) 